\title{
Effects of fasting in Ramadan on Glycemia, Lipid and other Metabolic profiles
}

Al Osali M., Al-Qanubi H., AlHashmi Z., AlBalushi A., Al Qassabi S., Al-Harthi S. Al Nahdha Hospital. Muscat. Oman

Background:Fasting during the month of Ramadan is a religious obligation, practiced by millions of Muslims around the world and during which Muslims refrain from food, liquids, and smoking from dawn to sunset. This type of fasting may have significant effects on lipid profile, Glycosylated Hemoglobin (A1c), Fasting Blood Sugar (FBS) and other metabolic and hematological indices ${ }^{1-4}$. An overall improvement in cardiovascular(CV) risk profile is noted during Ramadan fasting 1 .

Aim: To demonstrate the effect of fasting on total cholesterol(TC), LDL cholesterol(LDL), HDL cholesterol (HDL), Triglycerides (TG), A1c, FBS, urea, creatinine, uric acid, total protein, albumin, Complete Blood Count(CBC), and systolic and diastolic blood pressures (SBP \& DBP).

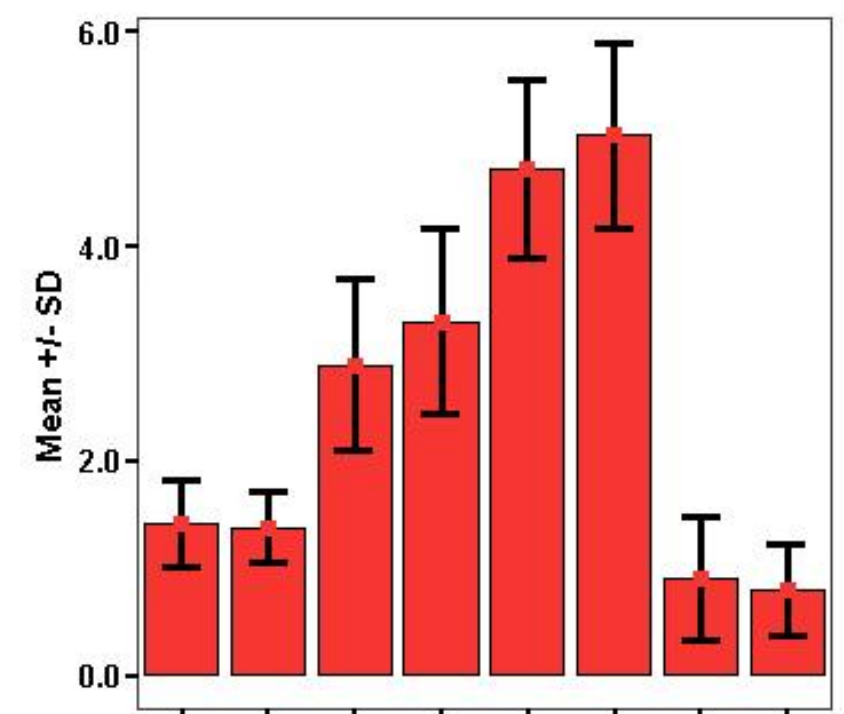

HDLA HDLB LDLA LDLB TCA TCB TGA TGB

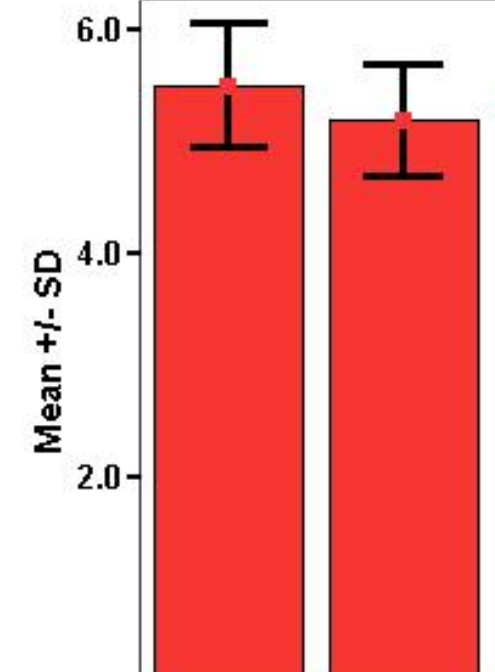

0.0 A1cB
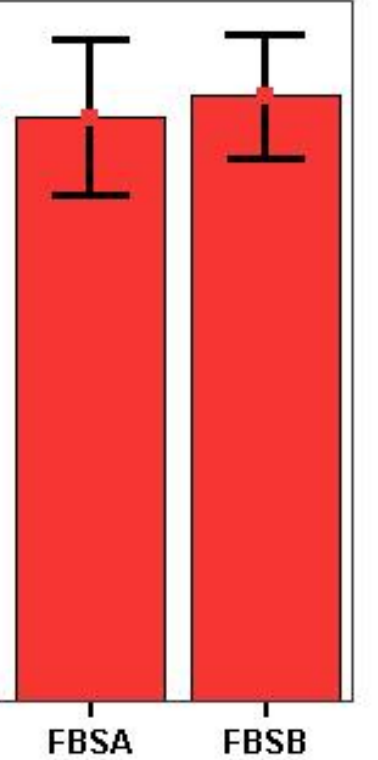

Discussion: Our study showed significant reduction of BMI, A1c, creatinine and TG and this could reflect weight reduction, better glycaemic control, and improvement in kidney function, and $\mathrm{CV}$ risk profile. However, there was significant increase in TC, LDL, and haematocrit which are risk factors for $\mathrm{CV}$ disease. The decrease in TG and increase in TC and LDL was also demonstrated in other studies ${ }^{3,5}$. In the contrary, some investigators reported reduction of TC, LDL, and TG and increase in HDL ${ }^{6-7}$. This conflict between studies could be due to ethnicity, gender distribution, fasting hours, seasonal differences, food habits, social and economic differences.

References

1.Mazidi M, et al. J Fasting Health. 2014; 2(2): 57-61.

2.Kamal S., et al. National Journal of Medical Research. 2012;2(4):407.

3. Saleh S.A. et al. Mal J Nutr.2005; 11(2): 143-150.

4.Al Hourani H.M., et al. Jordan Journal of biological sciences. 2009 ;2(3) $: 103$.

5.Pirsaheb S., et al. J Fasting Health. 2013; 1(1):6-12.

6.Shehab A. et al. PLoSOne.. 2012;7(10): e47615.

7.Quieq D. et al. Ann Saudi Med. 2002 ;22(5-6):297-9.

Keywords: Ramadan, fasting, A1c, HDL, LDL, TG.

Acknowledgements: Our gratitude to all staffs in the Department of

Internal Medicine, Al Nahdha Hospital for their contribution in the study.
Methods: Healthy individuals working in $\mathrm{Al}$ Nahdha Hospital and fasting Ramadan were included in the study. Pregnant ladies, and those who have chronic diseases, or using medications that can affect lipids or glucose levels are excluded. The individuals were reviewed in 2 visits, the $1^{\text {st }}$ was in the 5 days immediately preceding Ramadan and the $2^{\text {nd }}$ was in last 3 days of Ramadan. In both visits, the volunteers were subjected to Body Mass Index (BMI), and SBP \& DBP measurements and blood collection to check A1c, FBS, TC, LDL, TG, HDL, urea, creatinine, uric acid, total protein, albumin and CBC.

\begin{tabular}{|c|c|c|c|c|c|}
\hline & \multicolumn{2}{|c|}{ Before Fasting } & \multicolumn{2}{|c|}{ After Fasting } & \multirow[t]{2}{*}{ P value } \\
\hline & Mean & SD & Mean & SD & \\
\hline BMI & 27.96 & 7.37 & 27.28 & 7.37 & $<0.001$ \\
\hline TG & 0.90 & 0.58 & 0.79 & 0.42 & 0.040 \\
\hline A1c & 5.49 & 0.55 & 5.18 & 0.50 & $<0.001$ \\
\hline Creatinine & $\mathbf{5 5 . 5 7}$ & 11.96 & 51.54 & 12.05 & $<0.001$ \\
\hline TC & 4.71 & 0.83 & 5.03 & 0.86 & $<0.001$ \\
\hline LDL & 2.89 & 0.79 & 3.30 & 0.86 & $<0.001$ \\
\hline Hematocrit & 38.09 & 4.29 & 38.86 & 4.69 & 0.024 \\
\hline Total Proteins & 77.74 & 4.43 & 79.94 & 4.48 & $<0.001$ \\
\hline Albumin & 43.13 & 2.89 & 44.34 & 2.74 & $<0.001$ \\
\hline HDL & 1.41 & 0.41 & 1.37 & 0.34 & 0.384 \\
\hline Uric acid & 274.68 & 62.01 & 273.1 & 62.85 & 0.751 \\
\hline FBS & 5.23 & 0.70 & 5.42 & 0.55 & 0.110 \\
\hline
\end{tabular}

Results:47 individuals ( 7 males, and 40 females) were included in the study with a mean age 33 year (range 23-58). After fasting Ramadan, BMI, TG, A1c, and creatinine are significantly reduced and TC, LDL, total proteins, albumin and haematocrit are significantly increased. However, there were no significant changes in HDL, uric acid, urea, EBS, hemoglobin and SBP \& DBP.

Conclusion: The effect of Ramadan fasting on metabolic profile is variable. While, some parameters are significantly improved, others, are strikingly different. It positively affects glycaemic control and TG levels. A large study is advised, with standardization as regards ethnicity, fasting hours, gender distribution. and food habits.

Corresponding Author: Dr. Magdi Al Osali

Email:mag_alosali@yahoo.com.

The Authors have no conflict of interest 